\title{
Automatic TLI Recognition System Evaluation using AMPS Data
}

\author{
G. D. Lassahn \\ J. K. Partin \\ J. R. Davidson \\ HQ PROJECT MANAGER - Stephen Herrick \\ PROJECT NUMBER - ST474E
}

Published March 1995

Idaho National Engineering Laboratory Lockheed Idaho Technologies Company Idaho Falls, Idaho 83415

Prepared for the U.S. Department of Energy under DOE Idaho Field Office Contract DE-AC07-I

941013223 


\section{DISCLAIMER}

This report was prepared as an account of work sponsored by an agency of the United States Government. Neither the United States Government nor any agency thereof, nor any of their employees, make any warranty, express or implied, or assumes any legal liability or responsibility for the accuracy, completeness, or usefulness of any information, apparatus, product, or process disclosed, or represents that its use would not infringe privately owned rights. Reference herein to any specific commercial product, process, or service by trade name, trademark, manufacturer, or otherwise does not necessarily constitute or imply its endorsement, recommendation, or favoring by the United States Government or any agency thereof. The views and opinions of authors expressed herein do not necessarily state or reflect those of the United States Government or any agency thereof. 


\section{DISCLAIMER}

Portions of this document may be illegible in electronic image products. Images are produced from the best available original document. 


\begin{abstract}
This report describes some capabilities and limitations of the prototype automatic target recognition system ATR1, as determined by exercising the system with field-acquired AMPS data.
\end{abstract}




\section{SUMMARY}

\section{Capability}

We have developed an automatic target recognition system (ATR) which

* can be trained to find any kind of target

* allows fusion of image data from sensors of any type

* provides target identification unceitainty estimates

* uses small parallel processors to achieve high speed at low cost

* is compact enough to be used on board a reconnaissance aircraft

This ATR system can avoid errors by fatigued human analysts in scanning large amounts of data, and it can apply multi-image search algorithms that are too compicated to be effectively implemented by a human analyst.

\section{Accomplishments}

Previous reports (see references) demonstrate that this ATR system is versatile and can be adapted to a variety of applications. The current evaluation with the Airborne Multisensor Pod System (AMPS) Mission 4 data confirms the ability of this ATR system to handle real-world data with large, multiband images, and to effectively find targets of practical interest. The particular exercise reported here (finding gas effluent stacks) demonstrates that this ATR system is much more powerful than the standard image analysis systems that classify pixels according to trivial criteria or simply reformat two or three bands for display as a false color image.

In addition to having developed algorithms and software, we have designed and are acquiring two (different) prototype state-of-the-art hardware systems for evaluation and demonstration. One of these is already delivered, and the other should be delivered in a few days. Both systems are trivially expandable to achieve more speed.

\section{Near-Term Efforts}

In addition to the normal continuing enhancement of the system's software, we plan two specific activities that go beyond the basic system development:

(1) addition of automatic image registration, starting with the preliminary algorithms developed by Pacific Northwest Laboratory.

(2) adaptation of a copy of this ATR system for use with the gas effluent monitoring program at Lawrence Livermore National Laboratory.

\section{Long-Term Goals}

The advanced hardware with the versatile software we have developed comprises a very powerful tool for finding targets in image data. This siik system will be useful both for real-time scans and for very complicated off-line target searches. Potential applications include location of underground structures via thermal variations on the surface or via detection of gas effluents; scanning for signatures characteristic of rocket launches or of different types of explosions; and of course location of treaty-limited items in large amounts of surveillance data. This project is an excellent vehicle for research and development of multisensor data fusion techniques for nonproliferation and national security applications. 


\section{CONTENTS}

ABSTRACT $\ldots \ldots \ldots \ldots \ldots \ldots \ldots \ldots \ldots \ldots \ldots \ldots \ldots \ldots \ldots \ldots \ldots \ldots \ldots$

SUMMARY $\ldots \ldots \ldots \ldots \ldots \ldots \ldots \ldots \ldots \ldots \ldots \ldots \ldots \ldots \ldots \ldots$ iii

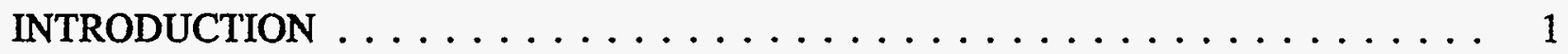

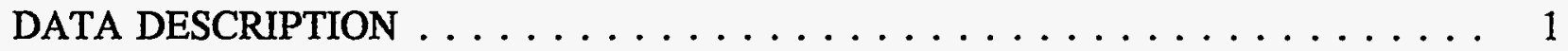

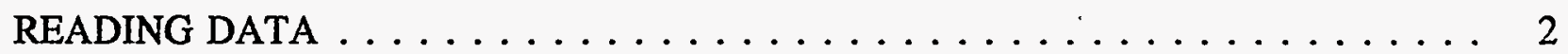

STACK SEARCH PROCEDURE $\ldots \ldots \ldots \ldots \ldots \ldots \ldots \ldots \ldots \ldots \ldots$

HANDLING LARGE IMAGES $\ldots \ldots \ldots \ldots \ldots \ldots \ldots \ldots \ldots \ldots$

RESULTS OF SEARCH FOR STACKS $\ldots \ldots \ldots \ldots \ldots \ldots \ldots$

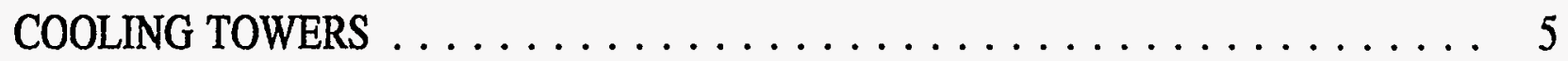

IMAGE REGISTRATION $\ldots \ldots \ldots \ldots \ldots \ldots \ldots \ldots \ldots \ldots$

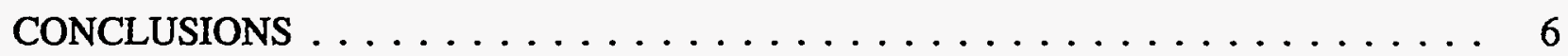

CONTINUING WORK $\ldots \ldots \ldots \ldots \ldots \ldots \ldots \ldots \ldots \ldots$

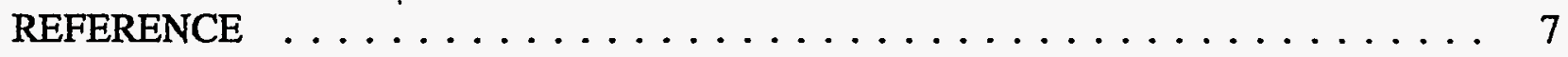




\section{Automatic TLI Recognition System Evaluation using AMPS Data}

\section{INTRODUCTION}

The purpose of this task is to develop an Automatic Treaty-Limited Item Recognition system, or an automatic target recognition (ATR) system. This task was started as part of the development of the Airborne Multisensor Pod System (AMPS), a reconnaissance system that can be attached to and carried aloft by any of several aircraft. This AMPS system includes several imaging sensors: a synthetic aperture radar (SAR), a Daedalus multispectral scanner, a CASI hyperspectral imaging system, a Barr \& Stroud IR18 Thermal Imager, a Wild Heerbrugg RC-20 large format camera, a Sony DXC-750 color video camera, and a COHU 5560 low-light video camera. Each scene of interest can be photographed by each sensor, so there can be several different images of each scene. The several images can be analyzed by a computer to determine the presence of previously specified objects of interest, or "targets", in the scene. The development of this computer system, an ATR system, is the present task. An earlier report ${ }^{1}$ describes the prototype ATR system, now named ATR1, and its evaluation and testing with other data. This current report describes new results and conclusions from testing ATR1 with real AMPS data.

\section{DATA DESCRIPTION}

The initial test of ATR1 was looking for nuclear reactors, and detectable indications of nuclear reactors might be cooling towers and gas effluent stacks. Therefore, we requested that AMPS Mission 4 include as a prime area of interest the Idaho National Engineering Laboratory (INEL) Test Reactor Area (TRA) which has both cooling towers and stacks, and that image data be acquired both before dawn, to make thermal infrared data useful, and during daylight, to make visible and near infrared data useful. We also requested data from other, secondary, areas of interest: the INEL chemical processing plant (CPP), which is of political interest because of it is an example of a facility with potential for producing nuclear weapons material; the INEL radioactive waste management complex (RWMC), which is of environmental interest from the viewpoint of containing hazardous wastes and is also of interest for learning how to remotely detect and characterize buried objects; and, two natural caves, which might test our ability to detect underground structures and are very interesting to anthropologists and naturalists.

Unfortunately, we were not able to acquire all of this data at INEL, because (1) the SAR data recording system malfunctioned and (2) smoke from a nearby forest fire made it unsafe for the AMPS aircraft to acquire the desired pre-dawn data, and schedules did not permit INEL data acquisition at another time. On the positive side, AMPS Mission 4 does include more complete imaging of several instances of cooling towers and effluent stacks at sites near Richland, Washington. 


\section{READING DATA}

The AMPS data immediately presented two challenges to ATR1: (1) The data tapes were in UNIX TAR format, which is a very common and reasonable choice but which was not compatible with the non-UNIX ATR1 system software; and (2) we had never before seen the data formats for the several imaging systems in the AMPS suite. It was necessary to learn about these formats and to augment the ATR software to allow reading data in these several formats, which presented no fundamental difficulty but did require some time. This expanded datareading capability is an essential improvement in the ATR1 software, made possible by the AMPS data availability.

\section{STACK SEARCH PROCEDURE}

Time limits did not allow a complete evaluation of all the available AMPS Mission 4 data in this study. We decided to devote most of our effort to the exercise of finding effluent stacks using multispectral scanner data.

As viewed from above with the AMPS multispectral scanners, at the minimum safe altitude of 1500 feet, an effluent stack is not at all distinctive or identifiable. However, if the sun is shining, a stack does cast a quite distinctive shadow in visible light and near infrared images. From knowledge of the range of sizes of interesting stacks, the latitude and longitude, the time of year and time of day, and the orientation and direction of the reconnaissance aircraft, we can predict the length, width, and direction of a stack shadow in an image. This combination was used as the primary criterion in this stack search exercise: we scanned the near infrared (1 micron) Daedalus image for dark straight line segments with specified minimum length (no maximum length was specified) and with width and angle in specified ranges. We will refer to the combination of length, width, and angle criteria as the morphological test.

Finding and characterizing a straight line segment in an image is very easy for a person's visual system, but surprisingly difficult for a computer. Even if the straight line segments are idealized, with high contrast, sharp edges, and no overlap with other objects in the image, identifying and characterizing them requires a substantial amount of computer calculation. If the straight line segment, the shadow, has slightly rough edges, because of rough terrain or simply because the image comprises discrete pixels; if different parts of the shadow have different contrast, because of varying brightness or reflectivity of the ground on which the shadow is cast; if the shadow crosses a building, a road, a ditch, a fence, or anything that either causes a gap in the stack shadow or adds an intersecting shadow of its own; these are realistic, expected factors that make stack shadow identification much more difficult for a computer, even though a person can very easily allow for these irregularities and decide with considerable confidence whether there is a stack shadow. As we learned by trial and error, identifying stack shadows by computer is a subtle process, fraught with nasty surprises that require additional computer processing to eliminate. The result is that the primary search, the morphological test, for stack shadows is quite time consuming, requiring about 200 seconds for a 500 by 500 pixel image with ATR1. In a real application, this might be unacceptably slow. It might be possible to develop 
different, faster software procedures to find shadows, and of course there is always the possibility of using a faster hardware system with more or faster parallel processors.

Figure 1 shows a preliminary result obtained during the development of the morphological test. In this image, there are two stacks, both of which cast very obvious shadows. The ATR program has found both shadows and drawn a loop around the maximum-contrast point in each. The program also identified as acceptable shadows five other features that are not really stack shadows. These features are actually dark lines (not completely clear in this report reproduction) with the correct sizes and orientations, representing dirt roads, earth embankments next to ponds, and other structures. There are two ways to discriminate between these spurious results and real stack shadows.

The first approach to eliminating the false positive results is to use narrower constraints on what length, width, and angle of shadow is acceptable. In this Daedalus data, there seems to be some distortion that causes stack shadows near the sides of the image to have an orientation slightly different from those at the center of the image. The obvious solution to this problem is to resample the image to remove such distortion, assuming that the distortion really is deterministic and predictable and that the shadows are big enough so that the required resampling does not excessively change the size and shape of the shadow. For the present exercise, we simply relaxed the tolerance on what shadow angles are acceptable, which of course allows more false positive results. Regardless of this image distortion problem, it will always be necessary to accept some greater-than-zero range of shadow angles, to allow for sloping terrain, uncorrectable aircraft orientation variations, uncertainties in the predicted shadow orientation due to uncertainties in time and position, and the discrete pixel nature of the image data. Similarly, the acceptance ranges of shadow length and width values must always be greater than zero, even if we decide that we are searching for stacks of one specific size and especially if we want to find all the stacks in a range of sizes. There will always be some other features that will fit the morphological test criteria we set for stack shadows, and the best we can do is make our tolerances as narrow as possible while still accepting all the stacks of interest.

The second method of discriminating between stack shadows and other structures is to do further analysis of all the positive results from the morphological test. In this exercise, we specified 7 features for the program to evaluate for each structure that was morphologically classified as a stack shadow: (1) the intensity in the shadow in the (slightly smoothed) 1 micron image; (2) the contrast between the shadow and its surrounding area in the 1 micron image; (3) the "brightness" parameter the program uses internally to describe how much the shadow looks like a straight line segment; (4) and (5) the intensity in the shadow in the 8 micron and the 12 micron images; (6) and (7) the products $(3)^{*}(2)$ and $(3)^{*}(1)$. The ATR system's training capability was used with some examples from the INEL TRA images to determine the optimal linear combination of these 7 features, and this linear combination was then used as the usual linear discriminant to distinguish between real stack shadows and other structures. The addition of this second step, which we will call the linear discriminant test, was successful in removing the false positive results in Figure 1 while still correctly accepting the real stack shadows.

There is a need for a special consideration when using the training process for the linear discriminant test after the morphological test. Normally, when forming training data, we would mark all of each stack shadow as target and we would mark broad areas of non-stack shadow as background. However, the linear discriminant test is to be applied only to the few pixels that 
are selected by the morphological test as being the highest contrast pixel in an acceptable shadow, no more than one pixel in each stack shadow. If the training process uses all the pixels we marked as background and target, the training is being based mostly on irrelevant pixels, pixels that have not passed the morphological test, and the results of this training process would not be expected to be very descriptive of the few relevant pixels. The training process script (the .fc file) must modify the mask so that only the relevant pixels are used in the optimization calculation. It may happen that the few pixels that are accepted by the morphological test are not sufficient for the training process. This requires that the set of training scenes be expanded, or that some reasonable pixels be added artificially, or that the linear discriminant test be recognized as redundant. The development of the technique of combining a non-trainable test (the morphological test) and a trainable test (the linear discriminant test) was enabled by having real-world AMPS data.

\section{HANDLING LARGE IMAGES}

Some of the AMPS Mission 4 images comprise more than 10 million pixels. Searching for stack shadows (probably a typical size application) requires memory allocation for 11 images the size of the raw input image, at 4 bytes per pixel. If the whole image were processed in one step, this would require more than 440 million bytes of memory (RAM), which is a rather large amount for a small computer. Even if we had this much memory, someone will always produce an image that is too large for whatever memory we have. We have long recognized this problem, and we have designed the software so that large images can be processed in parts, each part being small enough to fit in whatever memory is available. The AMPS data was the first real test of this capability, and it did indicate a need for some minor software modification which was immediately done. This test of handling large images is one more benefit of having AMPS data.

\section{RESULTS OF SEARCH FOR STACKS}

Part of the data from INEL TRA was used for training the linear discriminant test. The stack shadow search process, including both the morphological test and the linear discriminant test, was then applied to 13 Daedalus data sets from AMPS Mission 4:

INEL TRA, 4 passes at two different times of day

INEL CPP, 2 passes

INEL RWMC

INEL Middle Butte

INEL Moonshiner

WPPSS, 2 passes in daylight

ALE, 2 passes in daylight

To the best of our knowledge, the ATR system found all except 1 of the stacks in all this image data. The one known stack that was not found is at INEL CPP. There are two reasons, each. of which would be sufficient by itself, for the ATR system not finding this stack: (1) the stack 
is very large, wider than the maximum width we allowed in the search process; and (2) the stack shadow overlays numerous small structures which add their own shadows and change the simple linear stack shadow into an irregular shape that the program could not interpret as a line segment. The relevant part of the 1 micron image is shown in Figure 2, in which a smaller, correctly identified stack shadow is marked with a loop and the larger, missed stack shadow is about $8 \mathrm{~cm}$ below and to the left of the loop, and in Figure 3.

The stack search did also give some false positive results, some of which are not all bad in that they indicate the presence of things very similar to stacks. At TRA, there is a water tower, a tank supported at the top of four relatively thin legs and having a larger vertical pipe at the center. The pipe shadow, sometimes enhanced by the coincidental positioning of two leg shadows, is sometimes indicated as a stack shadow. Figure 4 shows part of one of the TRA scenes, with 3 real stack shadows marked. The water tower itself is hardly discernible, but the central column shadow and two leg shadows can be seen close to the left edge of the scene, halfway between the top and bottom edges. Figure 5 shows this area more clearly. The central column under the water tower seems like a reasonable object for the ATR system to interpret as a stack.

West of the WPPSS site is a structure which we believe is a large power line; in a good on-screen display of this image, both the conductors and their shadows are marginally visible. Figure 6 shows part of the 1 micron image of this scene. The ATR indicated two targets at this structure, which we assume are large poles or towers that support the power line. This also seems like a reasonable object for the ATR system to find in its search for stacks.

The ATR system also reported as targets some other structures that are clearly not targets. Figure 7 shows an agricultural area east of WPPSS, in which two non-stack structures (perhaps a canal and a dry ditch?) are marked as targets. Figure 8 shows the only reported target in the WPPSS area proper, which is believed to be a dirt road. Figure 9 shows part of an ALE scene with one false target. Marginally visible in this figure are shadows of some objects like power poles, too small to be identified as stacks, near the man-made structures at the bottom center of the picture. Figure 10 shows a dark area between two lighter dirt roads that was incorrectly indentified as a stack shadow. The very dark spot near the center of this figure is a depression at the entrance to Middle Butte Cave. There were a few other instances of false target identification, all nearly identical with the examples shown in these figures. As has been implied before, it is virtually impossible to eliminate all false target indications, in this or any ATR system.

\section{COOLING TOWERS}

Functioning cooling towers should radiate heat, and so should appear bright in thermal infrared images. This suggests the obvious and trivial analysis method in which we simply use - a linear combination of several images, some at thermal infrared wavelengths and some at shorter wavelengths. The training process in this ATR system would give optimal values for the linear combination coefficients and for the linear discriminant criterion. Data of this type should be taken at night, preferably just before dawn, so that objects which radiate because of stored heat acquired during the sunlit day would have cooled and would not appear nearly as bright as a true 
heat generator like a cooling tower. Unfortunately, it was not possible to acquire the requested pre-dawn data at the preferred INEL TRA location. We did try the above-mentioned analysis method on data acquired after 9:30 am at TRA; on the chance that the thermal emissions from the cooling towers might still be significantly stronger than the emissions from solar heated objects. We found that the situation was worse than expected, that the thermal emission data from the cooling towers had apparently saturated the imaging system (perhaps an expected result of trying to use what should be a night-time measurement in daylight) so that any intensity contrast between the cooling towers and other objects was decreased by some unknown amount. The results from this data varied from marginal to terrible, with the cooling towers being detected but with a large number of false positive target reports. When time permits, we will try the pre-dawn data from WPPSS, which should be much better.

\section{IMAGE REGISTRATION}

We have used only the Daedalus data from AMPS Mission 4. It would be at least interesting, perhaps emormously beneficial, to combine several different types of data in one analysis. However, the different types of data are in different formats, and there is not a pixelto-pixel correspondence between a Daedalus image and a SAR image, for example, of the same scene. That is, the images are not registered. Most image fusion processes, including the ATR system being developed in this project, require that the images be registered before any meaningful joint analysis can be done. Although it has never been an objective of this ATR project to develop image registration capabilities, we do have software of very limited capability which can do linear conversions of images to accomplish registration. However, using this simple software for large images such as the AMPS Mission 4 data would be very difficult. This lack of a reasonable capability for automatic or semi-automatic image registration is the reason for our not having done joint analyses on different types of AMPS Mission 4 data. Development of a good image registration scheme applicable to AMPS data is essential if we are to realize the full potential of AMPS or similar data.

\section{CONCLUSIONS}

The availability of AMPS Mission 4 data has allowed us to make significant improvements in the ATR system software in several areas, including (1) reading UNIX TAR data; (2) reading different AMPS imaging system data formats; and (3) processing large images in parts. It has also allowed us to learn about combining non-trainable and trainable target recognition procedures.

We found that the general structure of the ATR system is powerful and versatile and it can handle a wide variety of applications. Software enhancements, if necessary, can be done quite easily.

The stack-finding exercise demonstrates quite clearly the need for as much speed as possible. ATR1, our present prototype, is certainly adequate for simple operations like a linear combination/linear discriminant analysis, but there are probably many potential applications as 
computation-intensive as searching for stack shadows, and ATR1 may not be fast enough for some of these applications. We note that the ATR1 is substantially behind the state of the art in terms of speed and in terms of speed per dollar.

A major problem is lack of an ability to automatically register images with disparate data formats. This might be regarded as a problem separate from the ATR system, but it is at least closely related and must be solved to realize the potential of this ATR system.

\section{CONTINUING WORK}

This evaluation, along with discussions with other workers involved in the data fusion research effort in NN-20, have suggested two primary areas of work for the near future:

(1) Incorporate automatic image registration capabilities into this ATR system software. This will start with the image registration software now partly developed by Pacific Northwest Laboratory (Tom Lundeen). We will use what we can from the PNL software, add enough to make it a complete system, and adapt it to fit into our ATR system. This will allow easy use of the several disparate data types from AMPS. The automatic image registration software will be written in such a way that it can be expanded later to include whatever new data types are of interest.

(2) Coordinate with Lawrence Livermore National Laboratory (Ray Finucane, Joe Galkowski) to incorporate this ATR system into the LLNL stack effluent monitoring program. This will include acquiring computer hardware suitable for installation in an aircraft such as the AMPS aircraft; developing the software to interface directly with the sensor and control systems of interest, and improving our stack-finding algorithms.

Both of these tasks fit naturally into the existing overall plan for developing and deploying this ATR system, and these tasks will be done along with other tasks already specified in that plan.

\section{REFERENCE}

1. J. K. Partin, G. D. Lassahn, and J. R. Davidson, Automatic TLI Recognition System, Part 1: System Description and Part 2: User's Guide, EGG-EE-11298, May 1994.

2. G. D. Lassahn, Preliminary Evaluation of an ATR System Applied to Robotic Tank Inspection, EGG-EE-11040, October 1993. 


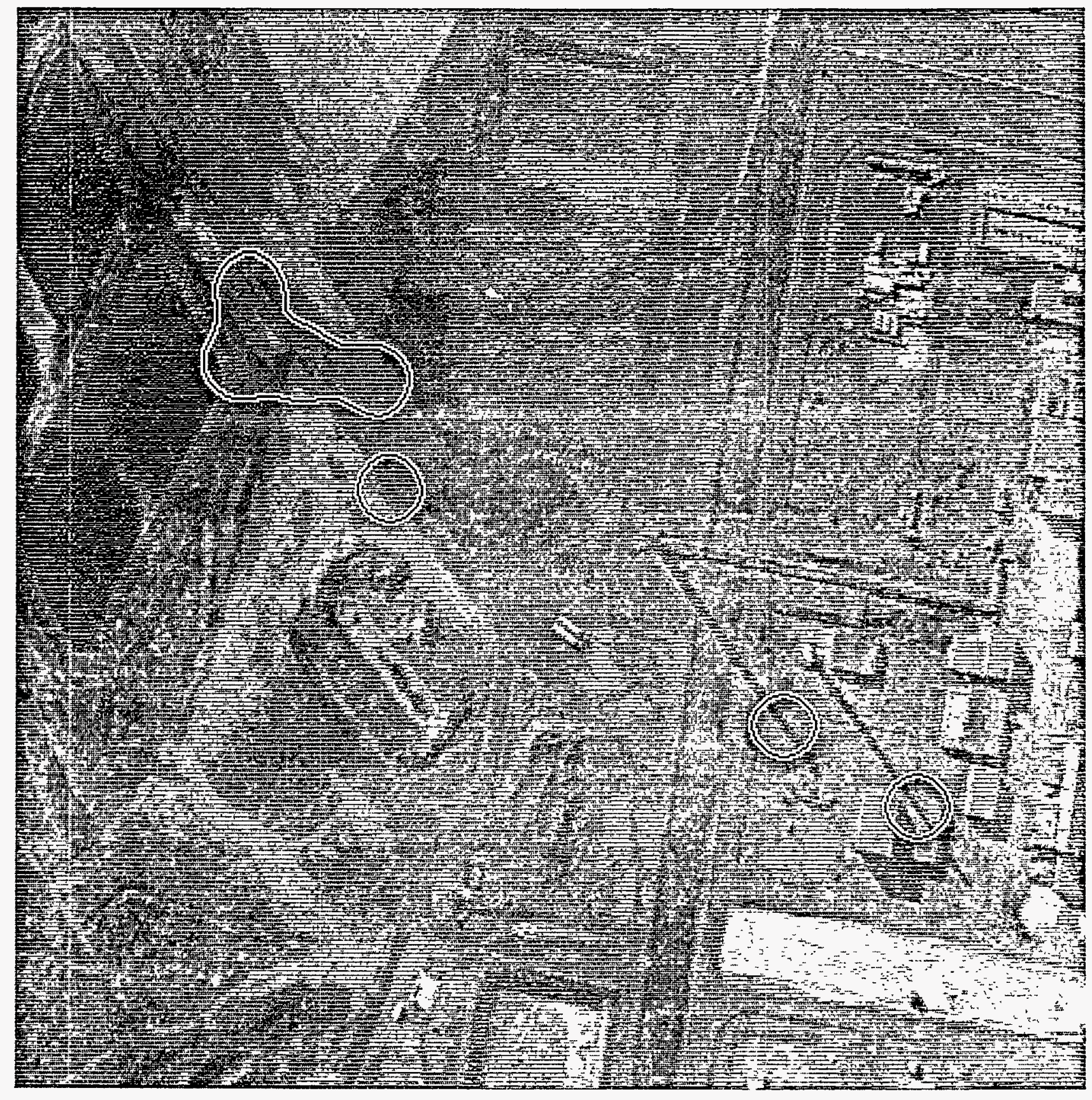

\section{IMAGE FILE: a:fd2606r2.ri}

Figure 1: Part of INEL TRA, showing two correctly identified targets and five false targets identified by a preliminary (incomplete) search for stack shadows. 


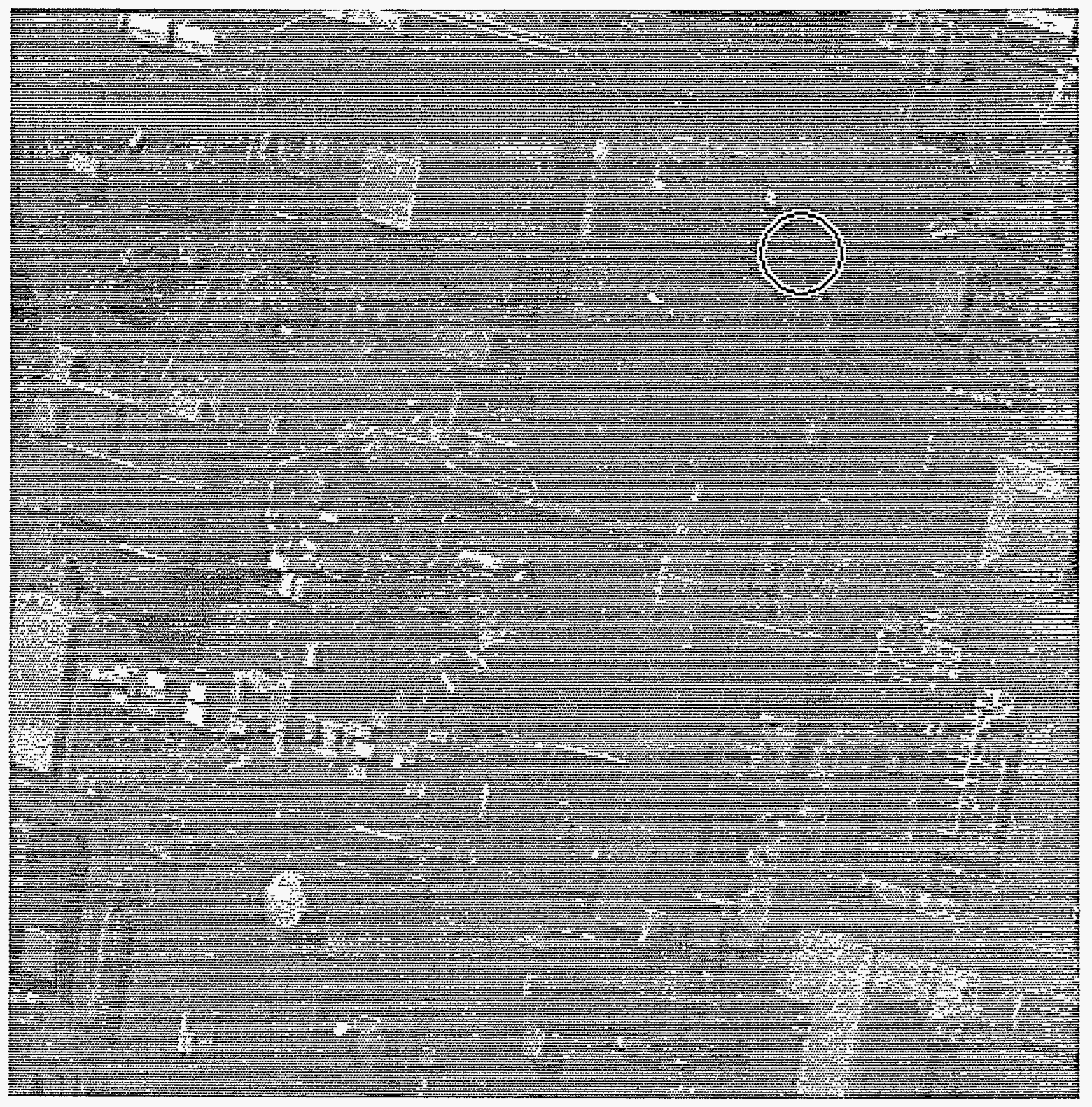

IMA

IMAGE FILE: a:ril3a.ri3

Figure 2: Part of INEL CPP, showing one correctly icinuified stack shadow and one justifiably missed larger stack shadow. 


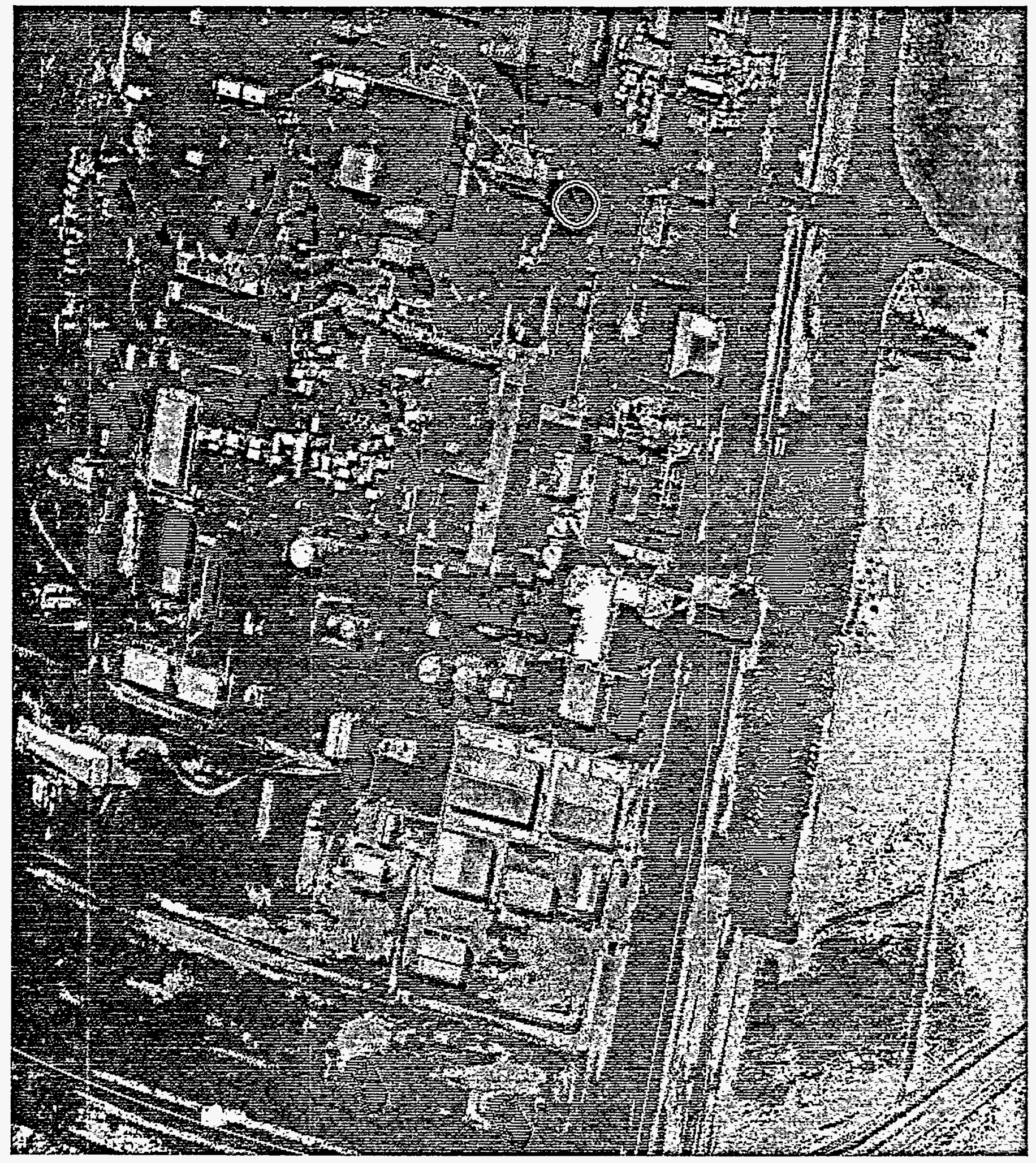

IMAGE FILE: a:ri000013.ri3

Figure 3: A broader view of the part of INEL CPP including the two stack shadows. 


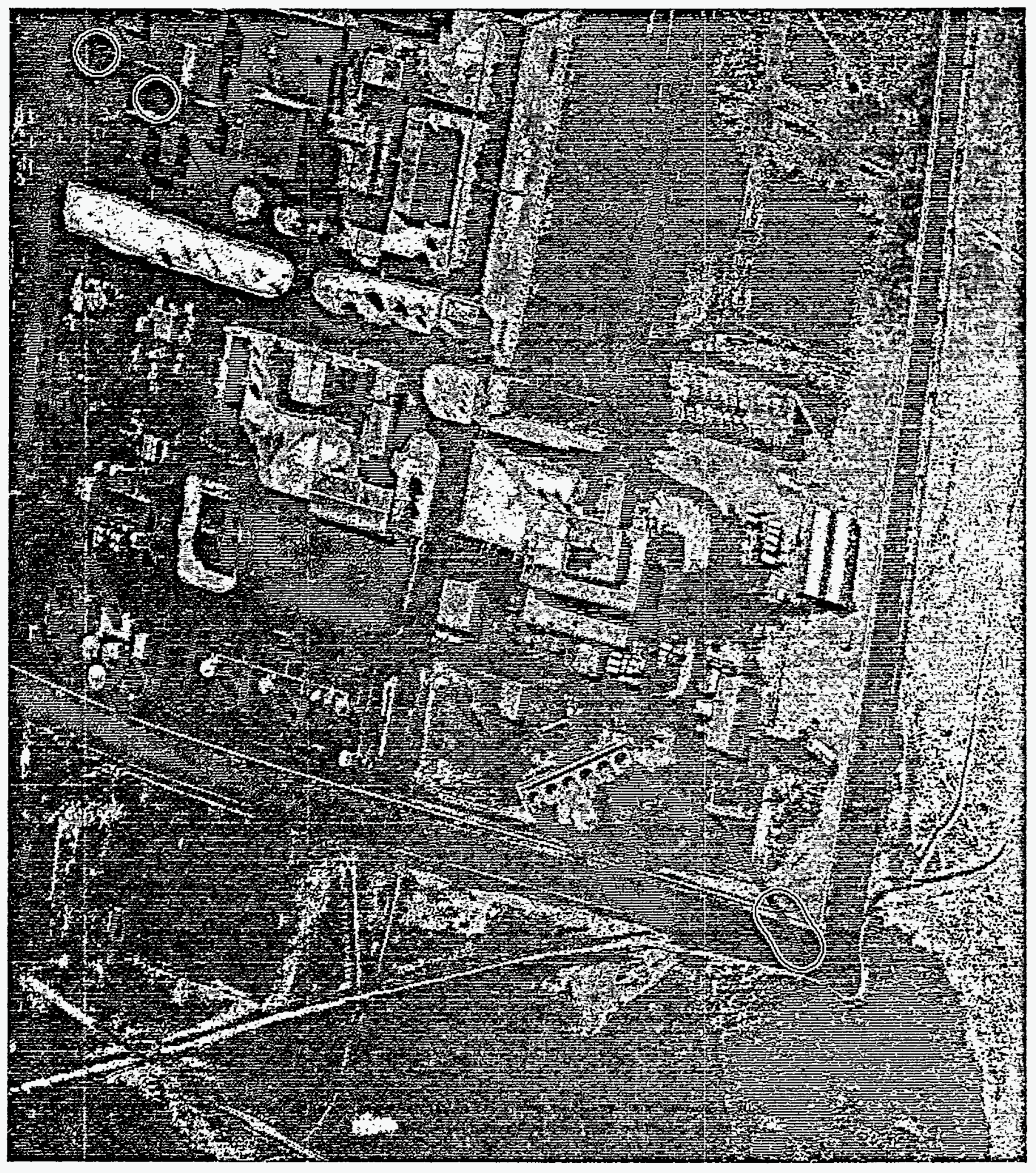

\section{IMAGE FILE: a:ri000011.ri3}

Figure 4: Three correctly identified stack shadows and one water tower at INEL TRA. 

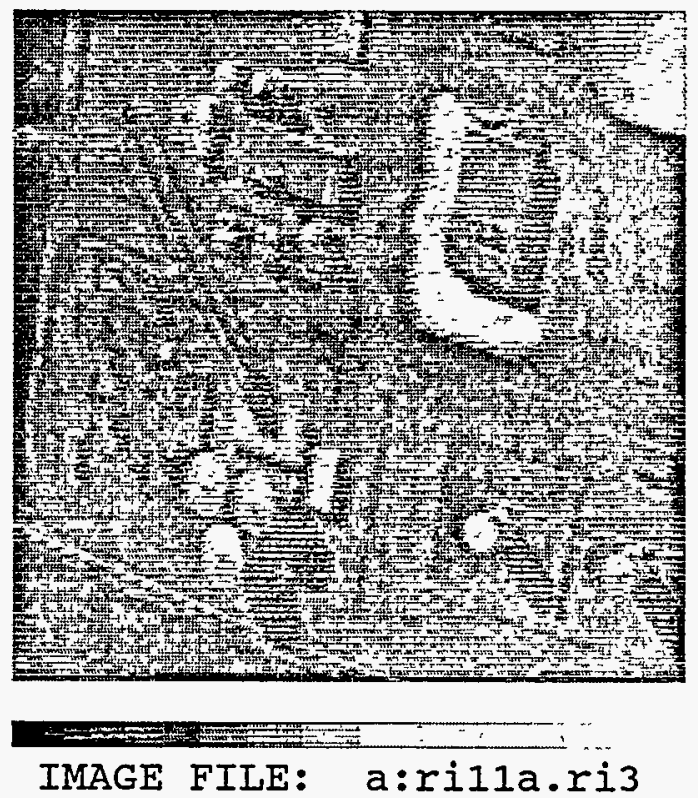

Figure 5: An enlarged view of the INEL TRA water tower. 


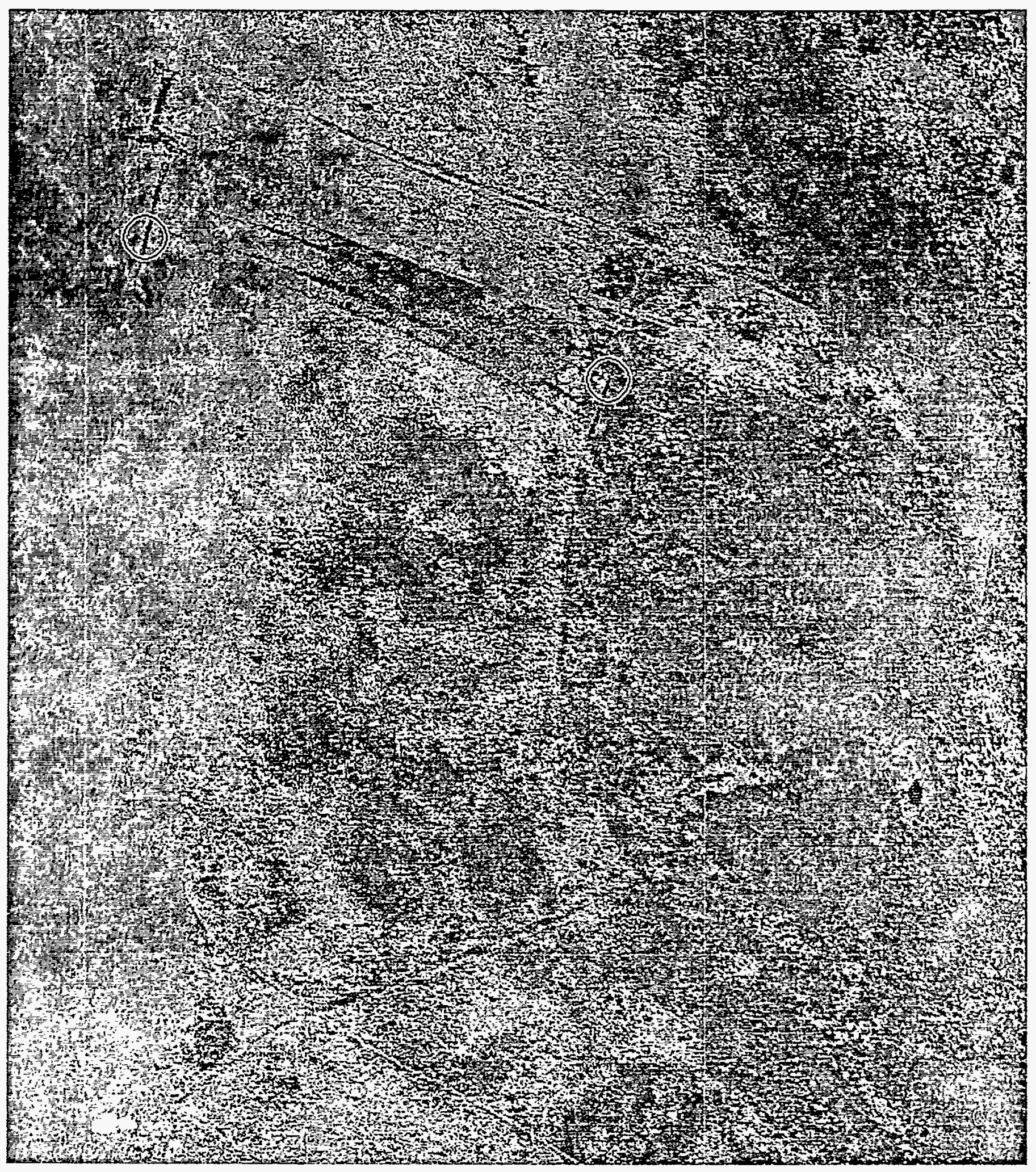

IMAGE FILE: a:ri000023.ri3

Figure 6: An area west of WPPSS, with two presumed power line towers identified as stacks. 


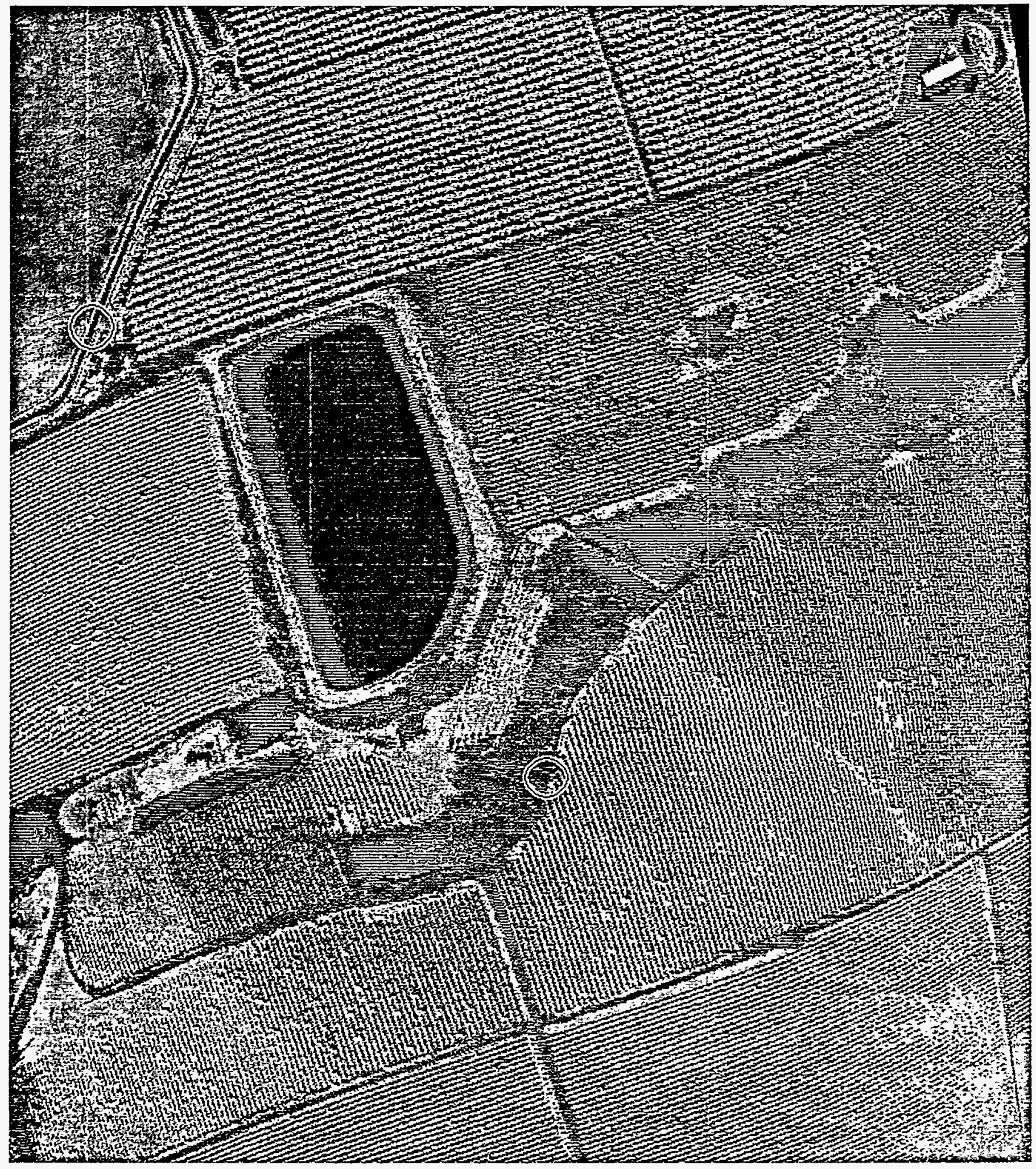

IMAGE FILE: a:ri000003.ri3

Figure 7: An agricultural area east of WPPSS, with two false indications of targets. 


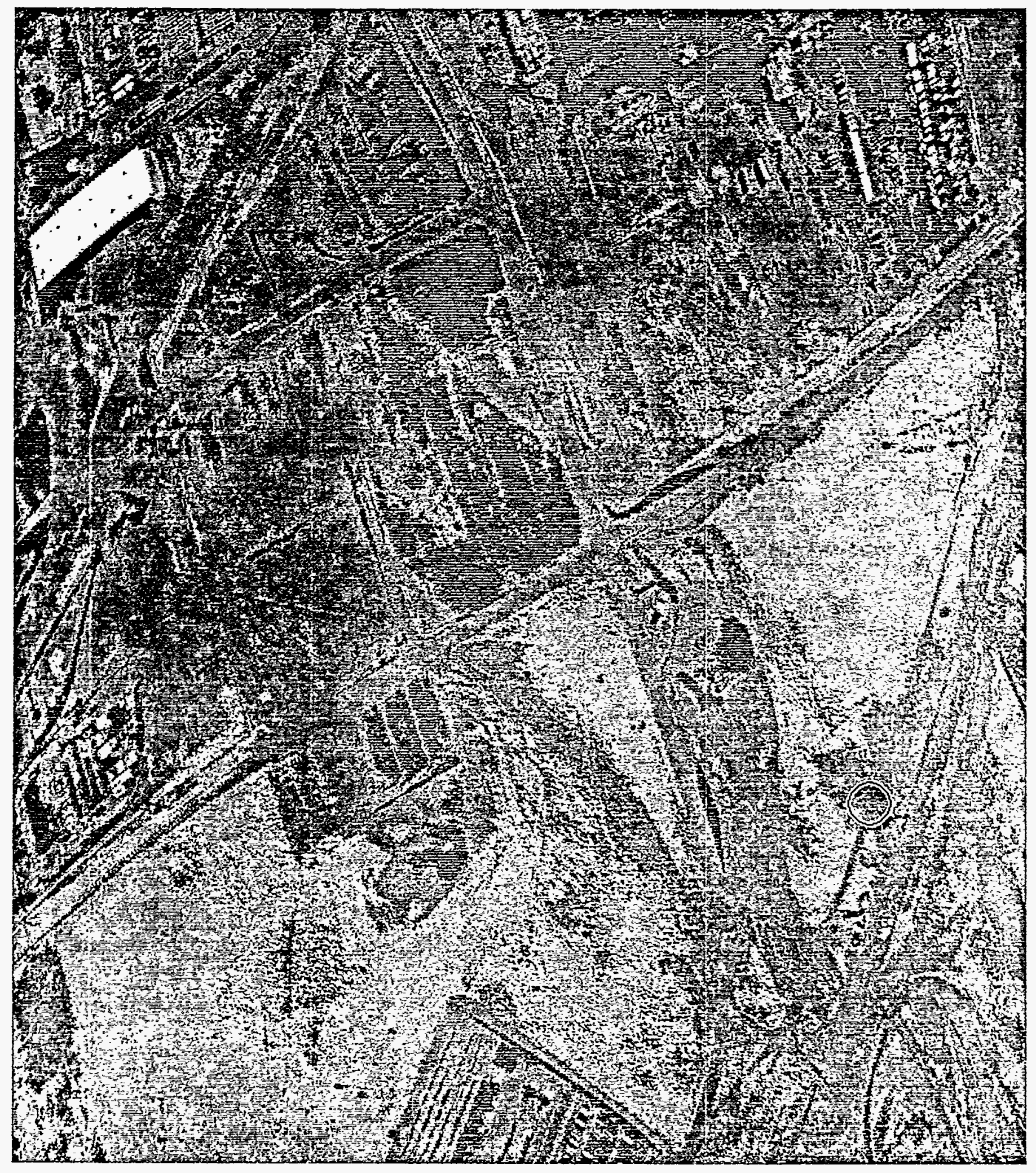

IMAGE FILE: a:ri000027.ri3

Figure 8: The only target indication in the WPPSS area, presumed to be a dirt road. 


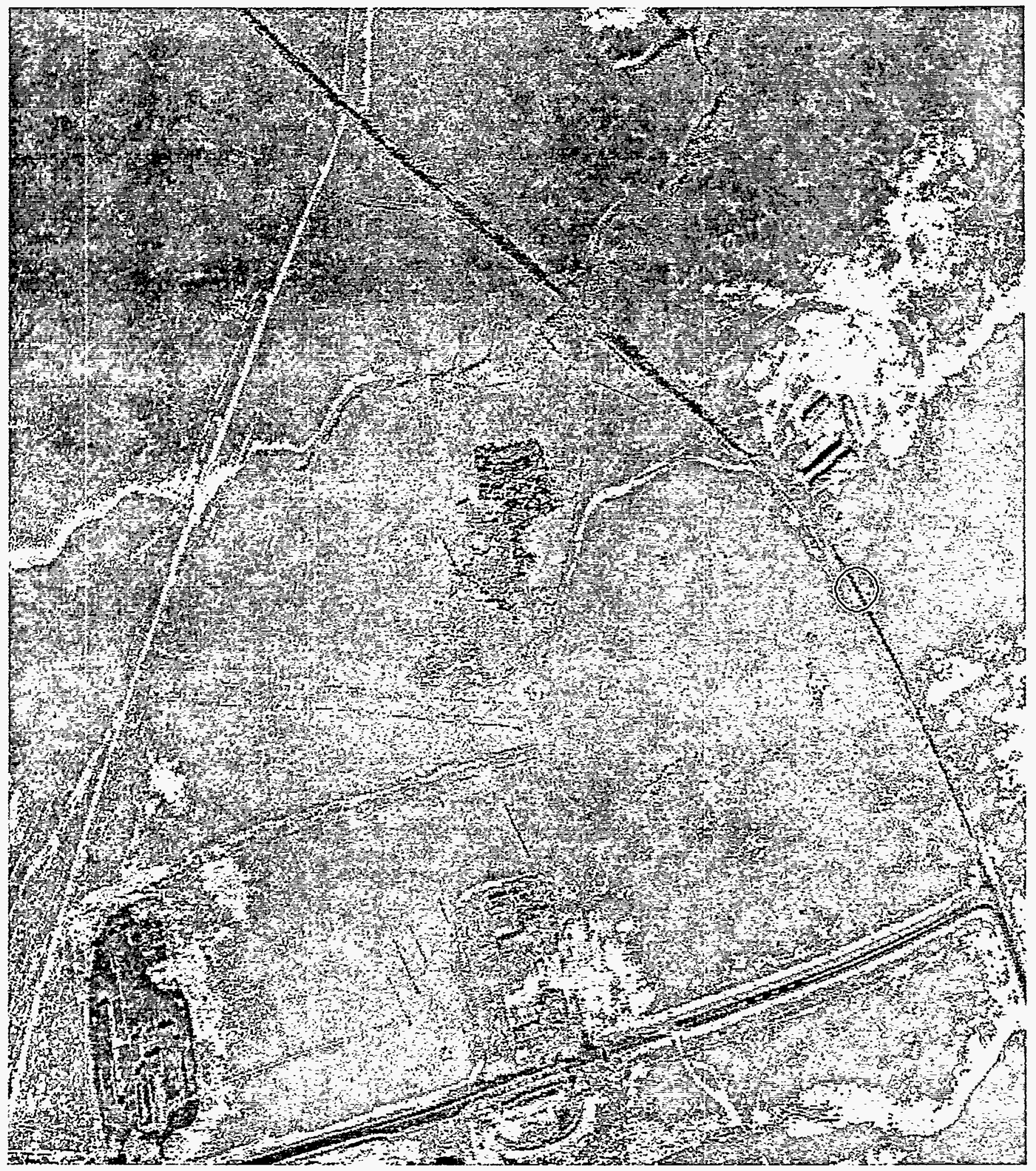

IMAGE FILE: a:ri000029.ri3

Figure 9: A false target indication near the Arid Land Ecology site (ALE). 


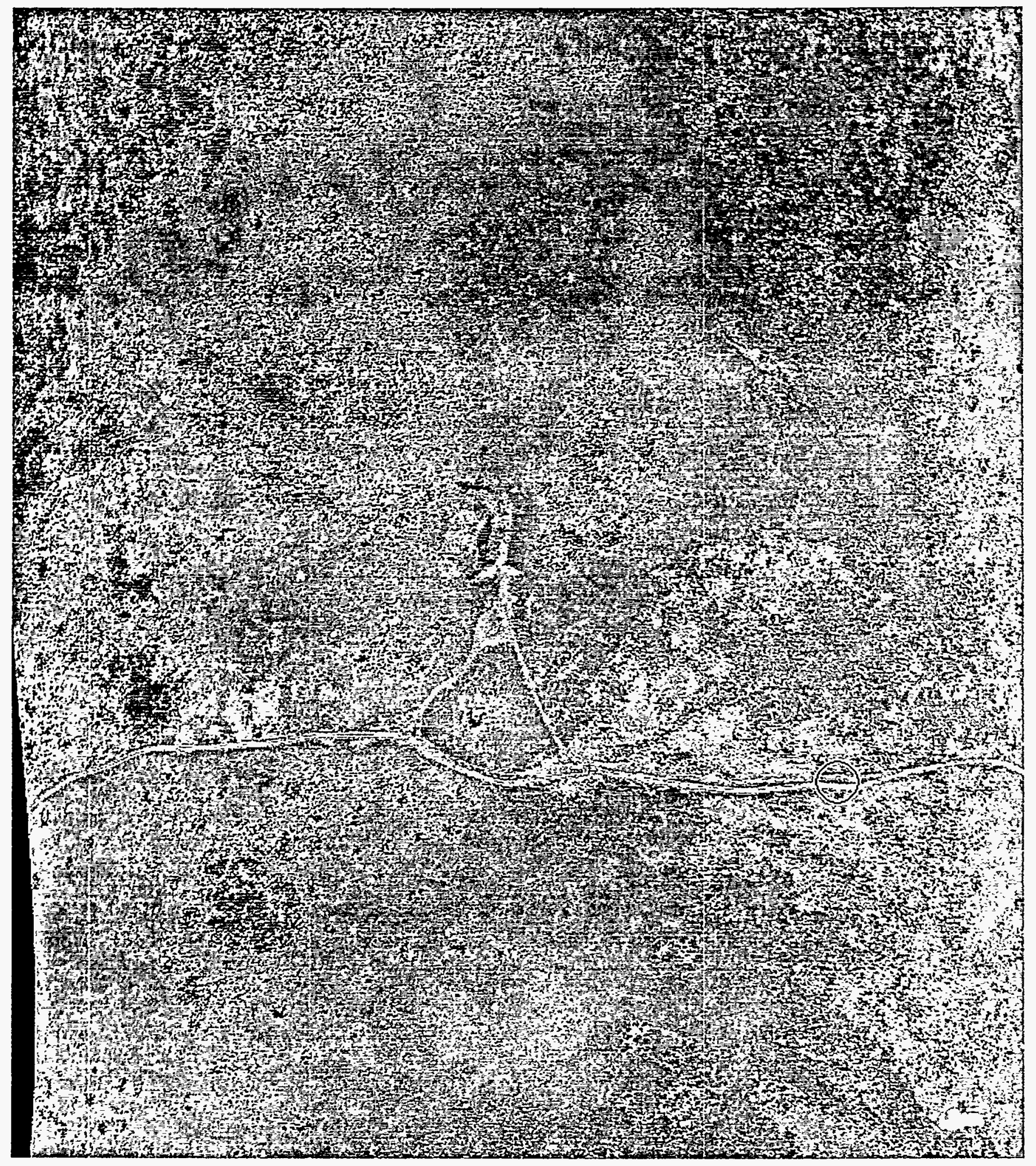

\section{IMAGE FILE: a:ri000015.ri3}

Figure 10: A false target indication near Middle Butte Cave. 